EEG revealed generalised encephalopathy and epileptic seizure predisposition on the right hemisphere, consistent with the clinical and radiological findings. Lumbar puncture was not immediately performed in view of the intracranial bleed and anti-coagulant treatment. Due to her poor clinical response, steroid treatment was started for suspicion of an autoimmune or inflammatory condition. A CT head done after 48 hours didn't show progression of the haemorrhage. We proceeded with lumbar puncture after withholding a dose of LMWH, screening for infection, metabolic and autoimmune panels. HSV-1 was detected in the CSF, despite no cutaneous or serological evidence of HSV infection. Concurrent cell counts showed pleocytosis confirming the clinical suspicion of encephalitis. She remained on aciclovir for 21 days as a definitive treatment.

Discussion Ischemic stroke and haemorrhage are increasingly recognised CNS manifestations of HSV infection. The brain ischemia is mostly related to multifocal cerebral large vessel vasculitis. HSV-related infarction is a rare but potentially treatable cause of stroke. Steroid treatment may be considered even in the absence of confirmation of vasculitis on neuroimaging. A systematic review showed up to $50 \%$ of cases with HSV ischemic manifestations presented with encephalitis while $30 \%$ presented with stroke-like symptoms.

Performing lumbar puncture is crucial to differentiate encephalitis from other forms of encephalopathy. Ideally it should be undertaken prior to initiation of aciclovir treatment, however this is usually delayed by other investigations, due to the nature of presentation. Therefore, high index of suspicion is needed to start aciclovir empirically as early treatment could reduce mortality and morbidity significantly.

Conclusion Cerebrovascular events in children should be recognised as a possible manifestation of HSV encephalitis. Aciclovir should be commenced early and CSF sampling should be undertaken at the earliest opportunity once safe to perform.

\section{COGNITIVE DISORDER IN CHILDREN WITH EPILEPSY}

Guzel Pilina*. Medical Center of Neurology 'Palladium'

10.1136/archdischild-2021-europaediatrics.389

Evaluation of different factors influence on cognitive function (CF) in children with epilepsy 20 children with epilepsy: 15 girls $(75 \%)$ and 5 boys (25\%), from 6 to 17 years old (mean age 11 years 9 months). The investigation included: standard neurology status, neuroimaging by CT or MRI, EEG, cognitive function examination by Epi Track Junior it was test. Anamnesis of Life and disease (duration and debut age of epilepsy, type and frequency of seizures, antiseizures therapy), delivery anamnesis and early motor and speech development.

There were several groups in Epi Track Junior results testing: good results $0 \%$, mild disorder of CF - 25\% (5 children), average disorder of CF - 20\% (4 children), severe disorder of CF - 55\% (11 children).

In light asphyxia (Apgar less than 7 degree) delivered 5 children (25\%), in the rest cases (75\%) Apgar was normal. Early development was normal in 19 children (95\%) and 1 child $(5 \%)$ with average speech retardation.
Unknown epilepsy etiology has 12 patients (60\%), genetic - $3(15 \%)$ and structural - 5 (25\%). Focal seizures without consciousness impartment were in 2 cases (10\%), generalized in $14(70 \%)$ and focal with secondary generalization in 4 $(20 \%)$. In remission were 3 children $(15 \%)$, with rare seizures were $9(45 \%), 1(5 \%)$ were with frequent seizures and 7 children were $(35 \%)$ with very frequent seizures.

All patients $(3(15 \%)$ in polytherapy had severe CF disorder. Children with frequent seizures (8 patients $(40 \%)$ had mild CF disorder in 2 cases (10\%), average CF disorder in 2 cases $(10 \%)$ and severe CF disorder in 4 cases $(20 \%)$.

Debut of seizures up to 7 years was in 9 children (45\%) and 7 (35\%) had severe CF disorder.

Duration of epilepsy from 1 to 3 years was in 4 children (20\%) and 2 children (10\%) of them had severe CF disorder. Duration epilepsy from 3 to 7 years were in 7 children (35\%) and all of them had severe CF disorder.

Severe and average CF disorder were showed in children with frequent seizures, early (up to 7 years) debut of seizures, duration of disease more than 3 years and polytherapy cases.

There was no influence on CF: Apgar score, early development, neurology deficit, structure changes in neuroimaging, type of epilepsy and seizures, EEG changes.

\section{EFFICACY OF GENE THERAPY WITH ELADOCAGENE EXUPARVOVEC IN PATIENTS WITH AADC DEFICIENCY COMPARED WITH NATURAL HISTORY CONTROLS}

Paul Wuh-Liang Hwu, Yin-Hsiu Chien, Ni-Chung Lee, Sheng-Hong Tseng, Antonia Wang, Jim Wang, Traci Schilling*, Sunay Ozdas, Chun-Hwei Tai. PTC Therapeutics, Inc

\subsection{6/archdischild-2021-europaediatrics.390}

Aromatic L-amino acid decarboxylase (AADC) deficiency is a rare autosomal recessive disorder resulting in marked or complete loss of dopamine, impeding normal motor development. Eladocagene exuparvovec, a recombinant adeno-associated virus containing the human cDNA encoding the AADC enzyme, is in clinical development for treatment of AADC deficiency.

Eladocagene exuparvovec was administered via bilateral infusion into the putamen of 28 children with AADC deficiency in 3 clinical trials (AADC-CU/1601 [8 patients, completed], AADC-010 [10 patients, ongoing], and AADC-011 [10 patients at data cutoff of 26 February 2020; ongoing]).

Patients received a total dose of $1.8 \times 1011 \mathrm{vg}(\mathrm{n}=21)$ or $2.4 \times 1011 \mathrm{vg}(\mathrm{n}=7$; AADC-011). Motor milestone achievement was assessed using the Peabody Developmental Motor Scales, 2nd Edition. Improvements in motor function from treated patients in the full analysis set were compared to 49 subjects from a comprehensive Natural History Database (NHDB). This database was derived from a systematic review of literature reporting data from patients with AADC deficiency. From an original pool of 237 patients, 49 were chosen as matched natural history controls. These natural history controls were chosen because they met the criteria of unique patients with confirmed AADC deficiency who had not participated in clinical trials of eladocagene exuparvovec, had documented lack of motor milestone achievement, and had a similar disease phenotype to patients in clinical trials.

As early as 12 months after receiving eladocagene exuparvovec, $44 \%$ of patients achieved head control and $20 \%$ of 
subjects could sit unassisted. At 24 months after gene therapy, $64 \%$ of subjects achieved head control, 50\% could sit unassisted, and 18\% could stand with support. At 60 months after gene therapy, $75 \%$ of subjects achieved head control, $67 \%$ of subjects could sit unassisted, $25 \%$ of subjects could stand with support, and $8 \%$ subjects could walk with support. In contrast, only $4 \%$ of the subjects in the NHDB control group achieved key motor milestones $(\mathrm{P}<0.0001)$. These results demonstrate that the attainment of milestones is sequential in nature and that the percentage of patients achieving more advanced milestones (eg, standing with support, walking with assistance) increases over time.

The number of patients achieving full head control and sitting unassisted was significantly higher in patients treated with eladocagene exuparvovec compared to the NHDB control group. The results indicate that patients with AADC deficiency treated with eladocagene exuparvovec show significant improvements in achieving motor milestones, impacting the natural history of disease.

\section{FEBRILE CONVULSIONS AND INFLUENZA A OR B- ARE THERE DIFFERENCES?}

Monika Kukuruzovic*

\subsection{6/archdischild-2021-europaediatrics.391}

Influenza viruses may cause predominantly respiratory illness, but could also be responsible for numerous neurologic symptoms and complications. All children who are admitted in hospital with symptoms and changes in their neurological status, especially during the flu season, should be tested even if their respiratory problems are mild or non-existent. The most common neurological complications are febrile convulsions.

In this study, we retrospectively reviewed patients with febrile seizures over two periods from 2008-2011. and 2018.2020. during a flu season (November-March) who had a proven influenza $A$ or $B$ virus and who were hospitalized at the Department of Neuropediatrics of the UHC Sestre milosrdnice. We aimed to find out if there were differences in the type of influenza depending on the period and what features of febrile convulsions were associated with influenza type A or type $\mathrm{B}$ and to emphasize the need for optimized prevention.

In the period 2008-2011. at our Department there were 480 patients hospitalized, 99 of them with febrile seizures. In the period 2017.-2020.

(end of February) there were 659 patients, 102 of whom with febrile seizures. The first period had 37 influenza positive patients (15 patients influenza type A, 5 influenza type B, 7 unknown) and the second period had 44 patients with proven influenza (36 patients had influenza type A, 8 patients had influenza type B).

In hospitalized children during both periods, influenza type A was associated with a higher incidence of febrile seizures than influenza type $\mathrm{B}$. The reason could be that influenza type $\mathrm{A}$ is more neurotropic than influenza type $\mathrm{B}$ and more often causes febrile seizures. Our finding of similar incidence of febrile seizures during both periods can be explained by continued poor prevention and fear of vaccination. This study indicates that there is a need to raise awareness of better prevention of influenza virus transmission.

\section{HEREDITARY NEUROPATHY WITH LIABILITY TO PRESSURE PALSY}

Iva Šeparović*, M Kukuruzović, M Malenica. UHC sestre milosrdnice

\subsection{6/archdischild-2021-europaediatrics.392}

A Case Report I.Šeparovićn ${ }^{1}$, M.Kukuruzović ${ }^{1}$, M.Malenica $^{1}$ 1.UHC Sestre milosrdnice, Zagreb, Croatia Hereditary neuropathy with liability to pressure palsies (HNPP) is a rare, autosomal dominant disease, with prevalence estimated between 0.84 and 16 per 100,000, affecting peripheral myelin, which manifests as recurrent, and usually transient, painless motor and/or sensory neuropathies. Neuropathies are triggered by minor trauma or repetitive movements with compression and traction of peripheral nerves. The most frequent forms of presentation affect the peroneal and the ulnar nerves. Brachial plexopathy occurs in 11\%-20\% and is an unusual clinical manifestation; bilateral presentation is even rarer. Genetic tests are available to aid in diagnosis as molecular analysis has identified a deletion in the chromosome $17 \mathrm{p} 11.2$ in the majority of these patients. The deletion of chromosome $17 \mathrm{p} 11.2$ in hereditary neuropathy with liability to pressure palsies appears to be the reciprocal meiosis product of the $17 \mathrm{p} 11.2$ duplication seen in Charcot-Marie-Tooth disease Type 1a. We present the clinical case of a 16-year-old boy, football player. It was his first episode, HNPP presenting with foot drop and palsy both nerves peroneus after distortion articulation talocruralis.

Electromyography studies showed subacute axonal lesions of both nerves peroneus. The diagnosis of hereditary neuropathy with liability to pressure palsy was confirmed by PMP22 deletion of chromosome $17 \mathrm{p} 11.2$. He started motor rehabilitation and avoidance of stressing factors with progressive recovery. These palsies generally resolve without surgical intervention but orthopedist decided to make decompression of nerve. The recovery followed quickly. After one-year follow up, he was completely asymptomatic. A case report is presented of a patient with this disorder to promote awareness and recognition that this entity should be considered in patients with multiple nerve palsies.

\section{NEUROLOGICAL PRESENTATION OF WILSON'S DISEASE IN A PEDIATRIC PATIENT WITH SILENT CIRRHOSIS}

Maša Malenica*, Orjena Žaja, Marina Mataia, Tomislav Gregurić, Monika Kukuruzović, Iva Šeparović, Barbara Perše, Ana Kovačević. UHC Sestre milosrdnice

\subsection{6/archdischild-2021-europaediatrics.393}

A previously healthy 13-year-old girl with a 5-month-history of hypersalivation, dysarthria, tremor, thrombocytopenia, and leukopenia was admitted to our hospital. On examination we noticed hypersalivation with an incomplete closing of the mouth, dysarthria, splenomegaly, resting and action tremor of the upper extremities, and slightly weakened hand grip. Jaundice, palmar erythema, or spider-like nevus were not present. Her body mass index was in the 1 st percentile (Z-score 2.21). Magnetic resonance (MRI) of the brain showed abnormal T2 hyperintensity in the basal ganglia, mesencephalon, and pons. Abdominal ultrasound indicated diffuse changes in liver parenchyma with circular edges, regenerative nodes, splenomegaly, and suspected portal hypertension, without ascites. Fibrosis was confirmed by liver fibroscan and abdominal MRI, which corresponded to laboratory findings (lower prothrombin 